\title{
Weight changes in postmenopausal breast cancer survivors over 2 years of endocrine therapy: a retrospective chart review
}

\author{
Kirsten A. Nyrop ${ }^{1,5} \cdot$ Allison M. Deal $^{1} \cdot$ Jordan T. Lee $^{2} \cdot$ Hyman B. Muss $^{1,5}$ • \\ Seul Ki Choi ${ }^{3} \cdot$ Samara Dixon $^{1} \cdot$ Amy Wheless $^{1} \cdot$ Lisa A. Carey ${ }^{1,5}$. \\ Shlomit S. Shachar ${ }^{4}$
}

Received: 28 October 2016/Accepted: 6 January 2017/Published online: 2 February 2017

\begin{abstract}
Purpose Obesity and weight gain after breast cancer (BC) diagnosis can affect cancer outcomes. This study explores the question of weight change during the first 2 years of endocrine treatment (ET) to identify the independent effects of $\mathrm{BC}$ diagnosis and treatment on post-diagnosis weight trajectories in early-stage postmenopausal BC survivors.

Methods The study design is a retrospective chart review. Chi square tests and ANOVA were used to compare patients who gained $>2 \mathrm{~kg}$, lost $>2 \mathrm{~kg}$, or had stable weight. Logbinomial regression models were used to evaluate associations between patient characteristics and weight trajectories. Results The final sample is $N=300$, with mean age at BC diagnosis of 65 years and $76 \%$ white. After 2 years of ET, $39 \%$ of study participants had gained $>2 \mathrm{~kg}, 27 \%$ had lost $>2 \mathrm{~kg}$, and $34 \%$ had stable weight. Relative risks (RR) for weight gain were as follows: age at diagnosis $=0.98(0.96$,
\end{abstract}

The abstract for this study was accepted for publication, 2016 ASCO Annual Meeting.

Kirsten A. Nyrop

kirsten_nyrop@med.unc.edu

1 Lineberger Comprehensive Cancer Center, University of North Carolina at Chapel Hill, Chapel Hill, NC, USA

2 Department of Exercise and Sport Science, University of North Carolina at Chapel Hill, Chapel Hill, NC, USA

3 Department of Health Behavior, Gillings School of Global Public Health, University of North Carolina at Chapel Hill, Chapel Hill, NC, USA

4 Oncology Institute, Rambam Health Care Campus, Haifa, Israel

5 Division of Hematology-Oncology, University of North Carolina at Chapel Hill, 170 Manning Drive, Campus Box 7305, Chapel Hill, NC 27599-7305, USA
0.99), being married $=1.48(1.04,2.12)$, weight change between BC diagnosis and start of ET $=0.98(0.97,0.99)$, Stage II $=1.42(1.01,2.01)$ or Stage III $=1.99(1.41$, 2.82), PR negative $=0.70 \quad(0.51,0.96)$, HER2 positive $=1.51(1.07,2.13)$, mastectomy $=1.49(1.12,1.98)$, axillary node dissection $=1.67(1.27,2.20)$, adjuvant chemotherapy $=1.49 \quad(1.02,2.19)$, and neoadjuvant chemotherapy $=2.29(1.67,3.14)$. Type of ET (tamoxifen or aromatase inhibitor) was not significant.

Conclusions In our sample of postmenopausal early-stage BC survivors, a majority had stable or lost weight during the first 2 years of ET. Higher disease complexity and associated treatment posed higher RR for weight gain.

Keywords Early stage breast cancer - Postmenopausal . Weight trajectories - Endocrine treatment - Chemotherapy

\section{Introduction}

Obesity, body composition, and energy balance have emerged as central concerns in cancer risk, prognosis, and survivorship [1-5], and as high priorities in oncology research [6-8]. This concern is reflected in clinical practice guidelines that emphasize attention to weight and energy balance through physical activity in cancer care [2, 4, 9-11]. In breast cancer (BC), a majority of women are overweight or obese at diagnosis $[12,13]$, with evidence linking risk for $\mathrm{BC}$ diagnosis with above normal weight especially strong in postmenopausal women [14-19].

\section{Implications of weight gain after $\mathrm{BC}$ diagnosis}

In addition to being a risk factor for $\mathrm{BC}$, obesity at $\mathrm{BC}$ diagnosis and weight gain after diagnosis have implications 
for prognosis, survival, and quality of life, again with evidence of adverse outcomes strongest in postmenopausal women. For example, high body mass index (BMI) at diagnosis has been associated with increased risk for $\mathrm{BC}$ mortality, with one study attributing $30-50 \%$ of BC deaths among postmenopausal women to high BMI at diagnosis [20]. Further, in a pooled analysis of 5675 estrogen receptor-positive BC survivors, $73 \%$ of whom were postmenopausal, the hazard ratio for late recurrence ( $\geq 5$ years) was significant for women who experienced $>10 \%$ pre- to post-diagnosis weight change (HR 1.24, 95\% CI 1.00-1.53); it was also significant for BMI in the range of 30 to $<35$ at 2 years of post-diagnosis (HR $1.40,95 \% \mathrm{CI}$ 1.05-1.86) [21]. In another study, where $68 \%$ of the women were postmenopausal at BC diagnosis, survivors who gained $>10 \%$ after diagnosis had worse all-cause mortality (HR 2.67, 95\% CI 1.37-5.05) compared to women who maintained their pre-diagnosis weight (plus or minus 5\% weight change), with a trend towards higher mortality when weight gain occurred within the first 2 years of diagnosis (HR 5.87, 95\% CI 0.87-47.8) as compared to later (HR 1.49, 95\% CI 0.85-2.57) [22]. That study also found that $>10 \%$ weight gain after diagnosis was associated with worse BC-specific mortality (HR 2.84, 95\% CI 1.15-6.65) [22]. A meta-analysis of postmenopausal BC survivors found a $15 \%$ increased risk for overall mortality (HR 1.15, 95\% CI 1.06-1.26) and 38\% increased risk for BC-specific mortality (HR 1.38, 95\% CI 1.11-1.71) in obese as compared to non-obese BC survivors [23].

\section{The role of endocrine treatment in weight gain in postmenopausal breast cancer survivors}

Receipt of chemotherapy has been linked to weight gain since the 1970s in both pre- and postmenopausal survivors [24-26]. The question arises whether other BC treatments are associated with weight gain. In postmenopausal women, most BC tumors (60-80\%) are hormone receptor (HR)-positive [27-32] and discovered at an early, highly curable stage [33, 34]. For postmenopausal women with this diagnosis, clinical guidelines recommend that adjuvant therapy includes endocrine treatment (ET) for at least 5 years to dramatically improve their overall prognosis and survival [35-38]. ET options are an aromatase inhibitor (AI) (anastrozole, letrozole, or exemestane) or selective estrogen receptor moderator (tamoxifen). For women on an AI, weight gain is of particular concern because it may exacerbate the common side effect of AI-associated arthralgia (joint pain, stiffness, or achiness) [39]. Weight gain may also be factor in ET treatment discontinuation [40-43] and may affect the efficacy of AI therapy [44-48]. Post-diagnosis weight gain in postmenopausal BC survivors has also been associated with intensified hot flashes [49, 50], physical function limitations [51], and other strains on quality of life [52]. For all of these reasons, the potential for weight gain during or associated with ET is of concern to both BC survivors who are about to start ET and to their oncology providers.

\section{Need for further research}

To identify the independent association of ET with weight gain and potential risk factors for weight gain during ET, our research team reviewed the literature from randomized controlled efficacy trials of ETs as well as observational studies of weight or weight change in BC survivors [53]. Specifically with regard to postmenopausal women, we found that proportions reporting weight gain as a "symptom" or bothersome symptom" ranged widely from 18 to $44 \%$ in Year 1 [54, 55] and from 7 to $55 \%$ in Year 5 [56, 57]. Among studies where weight in general and weight change over time were independently assessed by clinical or research staff, one study reported $>5 \mathrm{~kg}$ weight gain in $42 \%$ of women after 5 years on ET [58]. There was so much variation in the proportions of BC survivors who gained weight and in the amount of weight gain that it was not possible to ascertain definitive trends from the current evidence. Research gaps included specific and consistent timeframes (e.g., from BC diagnosis to a specific year of ET, or from start of ET to a specific year of ET), specific amounts of weight change (kilograms or pounds), and proportions who gained, lost, or remained stable (not just the proportion who gained or reported weight gain).

\section{Current study}

In light of the inconclusive evidence and to further explore the question of weight gain in postmenopausal BC survivors on $\mathrm{ET}$, we report here findings from a retrospective chart review focused on weight change during the first 2 years of ET. We focus on this timeframe because this is usually a period when BC survivors have regularly scheduled clinic visits with their oncology provider in order to monitor disease recurrence and ET side effects, including significant weight changes. Our specific focus is independently assessed weight measures $(\mathrm{kg})$ that are routinely taken by clinic staff during these visits, because these measures are readily accessible for oncology providers interested in monitoring weight changes in their patients. The first aim of our study is to describe weight trajectories in this patient population, with weight categorized as $\geq 2 \mathrm{~kg}$ weight gain, $\geq 2 \mathrm{~kg}$ loss, or stable weight (within $2 \mathrm{~kg}$ ). Our second aim is to describe BC tumor and treatment factors associated with weight change during the first 2 years of ET, with the specific objective of 
identifying the independent effect of each ET and of chemotherapy.

\section{Methods}

Data were derived from clinician notes and other medical personnel entries (such as nursing staffing assessed height and weight, and technician notes regarding chemotherapy and radiation) in the electronic medical records (EMR) of women seen in BC clinics of a university-affiliated tertiary hospital. To ensure best practice in retrospective chart review [59], the following procedures were followed: (1) research questions were well defined and clearly articulated a priori; (2) all variables used in our analysis had the same purpose as their original clinical intent (such as describing the $\mathrm{BC}$ diagnosis and treatment); (3) a standardized data abstraction form was used; (4) the abstractor was trained and monitored; (4) ambiguities in the data were resolved through discussion and consensus; (5) explicit inclusion and exclusion criteria were developed at the onset; and (6) confidentiality and ethical consideration were reviewed and approved by the Institutional Review Board of the University of North Carolina at Chapel Hill (IRB 15-1523).

\section{Study participants}

Female BC patients were identified on a consecutive basis from clinic schedules and from the Neoadjuvant Database at the University of North Carolina (UNC) Lineberger Comprehensive Cancer Center (LCCC9815, IRB 01-1154). To eliminate the known association of menopausal status with weight gain in BC survivors [60, 61], we limited our study to women who were postmenopausal at the time of their first BC diagnosis. Postmenopausal status was recorded in the clinician notes when a treatment plan that includes ET was presented to the patient and/or when initiation of ET was discussed with the patient. Clinicians state the patient's menopausal status as the reason for prescribing a specific ET, such as AI rather than tamoxifen, and sometimes also refer to lab tests to confirm menopausal status. BC survivors also had to meet the following eligibility criteria: stage 0-III BC diagnosis, on ET for at least 2 years, and weight data throughout 2 years of ET.

Women were excluded if their $\mathrm{BC}$ had recurred or metastasized within the timeframe of our study (BC diagnosis through 2 years of ET), as disease progression could have an independent effect on weight change (e.g., weight loss) [62]. BC survivors were also excluded if they had other non-cancer comorbidities described in clinician notes as poorly controlled or mobility impairments (such as being wheelchair bound) as these circumstances may have an independent effect on weight trajectories [63, 64]. Finally, women were excluded when clinician notes documented ET non-adherence or discontinuation, as ET dose may affect weight trajectories. Individual cases of inclusion or exclusion from the study were discussed and determined jointly by three authors (KAN, SSS, and JTL).

\section{Measures}

Variables selected for inclusion in the study were determined for their hypothesized potential association with weight trajectories and the quality and consistency of their availability in the EMR. All data were extracted from clinician notes with the exception of weight data which were recorded in the EMR by nursing staff. Data from the EMR were entered into a REDCap [65] database and included the following: (a) patient-reported age, race, ethnicity, marital status, and employment status at BC diagnosis; (b) BC diagnosis (stage, grade, nodal status, hormone receptor (HR) status, HER2 status); (c) BC treatment (surgery type, axillary surgery, chemotherapy, radiation); (d) ET history (including switching among ETs); (e) history of hormone replacement therapy (HRT); and (f) comorbidities at $\mathrm{BC}$ diagnosis. Height and weight were independently assessed by $\mathrm{BC}$ clinical staff at the time of diagnosis and at 3-4 month intervals for the first 2 years of ET, using scales located in the BC clinic and standardized procedures for measuring height and weight. The final weight measure was within 3 months of completing 2 years on ET. Data entry was conducted by one author (JTL) with training and quality control provided by three authors (AMD, SSS, and KAN). Completeness and accuracy of the data were achieved through cross-checking within the patient's medical record to resolve any inconsistencies in clinician entries.

\section{Statistical analysis}

Descriptive statistics were used to characterize the final sample and summarize findings based on three weight change classifications from the first 2 years on ET: weight loss $>2 \mathrm{~kg}$, stable weight plus or minus $2 \mathrm{~kg}$, and weight gain $>2 \mathrm{~kg}$. Among 300 participants, 38 had 24-month weight data imputed from weight data at 21 months. Percentages and means are compared between patients in the three weight change groups using Chi squared tests and ANOVA. Log-binomial regression models are used to evaluate univariate associations between patient characteristics and weight gain of more than $2 \mathrm{~kg}$, and relative risks (RR) are reported for all variables. All analyses were conducted using SAS v9.4 statistical software (Cary, NC). 


\section{Results}

\section{Study participant characteristics}

Characteristics of the final sample $(N=300)$ are presented in Table 1 (see Fig. 3 in Appendix for a flow diagram of $\mathrm{BC}$ survivors excluded from the final sample). Mean age at $\mathrm{BC}$ diagnosis was 65 years and the sample was predominantly white (76\%), non-Hispanic (98\%), married (64\%), and currently not employed (55\%). At the time of BC diagnosis, the mean BMI was 29 , with most participants categorized as overweight (28\%) or obese (39\%). None of the study participants were below normal weight at diagnosis. Mean number of comorbidities at $\mathrm{BC}$ diagnosis was 2.2, ranging from 0 to 6 . Eighty-two percent of tumors were Stage $0-\mathrm{II}$ and $71 \%$ were Grade 1 or 2 . BC surgery was $57 \%$ lumpectomy and $43 \%$ mastectomy. Most axillary surgery was axillary dissection (67\%). Forty-nine percent of study participants had chemotherapy and $68 \%$ had radiation. ET treatments were $38 \%$ anastrozole, $19 \%$ letrozole, $3 \%$ exemestane, $14 \%$ tamoxifen, $13 \%$ more than one AI, and $13 \%$ tamoxifen and AI. Mean time from BC diagnosis to start of ET was 0.46 years (SD 0.46), 0.26 (SD 0.21 ) for women who did not receive chemotherapy, and 0.66 years (SD 0.31) for those who received chemotherapy.
Table 1 Study participant characteristics $(N=300)$

\begin{tabular}{|c|c|}
\hline Variable & $\begin{array}{l}\text { Results } \\
\text { Mean (standard deviation) or \% }\end{array}$ \\
\hline \multicolumn{2}{|l|}{ At diagnosis } \\
\hline Age & $64.4($ SD 8.6$)$ (range 42.6-92.0) \\
\hline \multicolumn{2}{|l|}{ Race } \\
\hline White & 76 \\
\hline African American or other & 24 \\
\hline Ethnicity—non-Hispanic & 98 \\
\hline \multicolumn{2}{|l|}{ Marital status } \\
\hline Married & 64 \\
\hline Not married (single, separated, divorced, widowed) & 36 \\
\hline \multicolumn{2}{|l|}{ Employment status } \\
\hline Currently not employed & 55 \\
\hline Currently employed & 45 \\
\hline Body mass index (BMI) category & 29.3 (SD 6.7) (range 18.6-58.6) \\
\hline Normal $(18.5$ to $<25)$ & 33 \\
\hline Overweight $(25$ to $<30)$ & 28 \\
\hline Obese I $(30$ to $<35)$ & 21 \\
\hline Obese II $(\geq 35)$ & 18 \\
\hline Hormone replacement therapy & 57 \\
\hline Comorbidities & $2.2(\mathrm{SD} 1.6)($ range $0.0-6.0)$ \\
\hline $0-1$ & 34 \\
\hline $2-3$ & 46 \\
\hline $4+$ & 20 \\
\hline \multicolumn{2}{|l|}{$B C$ diagnosis } \\
\hline \multicolumn{2}{|l|}{ Stage } \\
\hline Stage 0 & 2 \\
\hline Stage I & 43 \\
\hline Stage II & 37 \\
\hline Stage III & 18 \\
\hline \multicolumn{2}{|l|}{ Grade } \\
\hline 1 & 27 \\
\hline 2 & 44 \\
\hline 3 & 29 \\
\hline Nodal status-positive & 39 \\
\hline ER positive & 98 \\
\hline PR positive & 84 \\
\hline
\end{tabular}


Table 1 continued

\begin{tabular}{|c|c|}
\hline Variable & $\begin{array}{l}\text { Results } \\
\text { Mean (standard deviation) or } \%\end{array}$ \\
\hline HER2 positive & 12 \\
\hline \multicolumn{2}{|l|}{ BC treatment } \\
\hline \multicolumn{2}{|l|}{ Surgery type } \\
\hline Lumpectomy & 57 \\
\hline Mastectomy & 43 \\
\hline \multicolumn{2}{|l|}{ Axillary surgery } \\
\hline Axillary dissection & 67 \\
\hline Sentinel biopsy & 33 \\
\hline \multicolumn{2}{|l|}{ Chemotherapy } \\
\hline Yes & 49 \\
\hline No & 51 \\
\hline \multicolumn{2}{|l|}{ Chemotherapy timing } \\
\hline Neoadjuvant & 51 \\
\hline Adjuvant & 49 \\
\hline \multicolumn{2}{|l|}{ Radiation } \\
\hline Yes & 68 \\
\hline No & 32 \\
\hline \multicolumn{2}{|l|}{ Endocrine treatment } \\
\hline Anastrozole only $(\text { Arimidex })^{\odot}$ & 38 \\
\hline Letrozole only (Femara) ${ }^{\odot}$ & 19 \\
\hline Exemestane only (Aromasin) ${ }^{\odot}$ & 3 \\
\hline Tamoxifen only & 14 \\
\hline More than one AI & 13 \\
\hline Tamoxifen and AI & 13 \\
\hline
\end{tabular}

$E T$ endocrine treatment, $E R$ estrogen receptor, $P R$ progesterone receptor, $H E R 2$ human epidermal growth factor receptor 2, $A I$ aromatase inhibitor

\section{Weight change during first 2 years of ET}

Associations between participant characteristics and weight change are presented in Table 2, with study participants grouped into one of the three categories according to their weight change at the end of 2 years on ET. Overall, $39 \%$ of study participants gained $>2 \mathrm{~kg}, 27 \%$ lost $\geq 2 \mathrm{~kg}$, and $34 \%$ had stable weight $( \pm 2 \mathrm{~kg})$. Weight gain and weight loss proportions in $2 \mathrm{~kg}$ increments are shown in Fig. 1. Figure 2 shows that women who were overweight (BMI 25-29.9) or obese class I (BMI 30-34.9) at BC diagnosis had the highest proportion of women who gained $>2 \mathrm{~kg}$ during ET, with more than $40 \%$ of the women in these two groups gaining $>2 \mathrm{~kg}$. In turn, women who were obese class II $(\mathrm{BMI} \geq 35)$ at $\mathrm{BC}$ diagnosis comprised the highest proportion of women who lost $>2 \mathrm{~kg}(46 \%)$ during 2 years of ET, and women who were normal weight at BC diagnosis had the highest proportion whose weight remained stable during ET (44\%). Patient characteristics significantly associated with weight gain in 2 years of ET were as follows: being married $(p=0.05)$, weight loss between diagnosis and start of ET $(p<0.01)$, BMI category at diagnosis $(p<0.01)$, higher tumor stage $(p<0.01)$, HER2-positive tumor $(p=0.03)$, mastectomy $(p<0.01)$, axillary node dissection $(p<0.01)$, and chemotherapy $(p<0.01)$.

\section{Unadjusted relative risk for weight gain}

In Table 3, we present the RR for gaining more than $2 \mathrm{~kg}$ during ET based on participant characteristics. Significant differences in risk $(95 \%$ confidence interval) were found for being married $\mathrm{RR}=1.48(1.04,2.12)(p=0.03)$, Stage II $\mathrm{RR}=1.42(1.01,2.01) \quad(p=0.04) \quad$ or $\quad$ III $\mathrm{RR}=1.99 \quad(1.41,2.82) \quad(p=0.0001), \quad$ HER2-positive $\mathrm{RR}=1.51 \quad(1.07, \quad 2.13) \quad(p=0.02), \quad$ mastectomy $\mathrm{RR}=1.49(1.12,1.98)(p=0.006)$, axillary node dissection $\mathrm{RR}=1.67 \quad(1.27,2.20) \quad(p=0.0002)$, adjuvant chemotherapy $\mathrm{RR}=1.49(1.02,2.19) \quad(p=0.04)$, and neoadjuvant chemotherapy $\mathrm{RR}=2.29 \quad(1.67, \quad 3.14)$ $(p<0.0001)$. Older women had a lower risk for weight gain during ET RR $=0.98(0.96,0.99)(p=0.002)$, as did women with Progesterone Receptor (PR)-positive tumors' $\mathrm{RR}=0.70(0.51,0.96)(p=0.03)$. Weight loss between 
Table 2 Associations between participant characteristics and weight change after 2 years on endocrine treatment $(\mathrm{ET})(N=300)$

\begin{tabular}{|c|c|c|c|c|}
\hline & \multicolumn{3}{|c|}{ Weight status after 2 years on endocrine treatment (ET) } & \multirow[t]{2}{*}{$p$ value } \\
\hline & $\begin{array}{l}>2 \mathrm{~kg} \text { weight loss } \\
(N=81,27 \%)\end{array}$ & $\begin{array}{l}\text { Stable weight } \\
(N=102,34 \%)\end{array}$ & $\begin{array}{l}>2 \mathrm{~kg} \text { weight gain } \\
(N=117,39 \%)\end{array}$ & \\
\hline \multirow[t]{2}{*}{ Age at diagnosis } & $65.1(\mathrm{SD} 9.2)$ & $66.1(\mathrm{SD} 8.7)$ & $62.5(\mathrm{SD} 7.8)$ & \multirow[t]{2}{*}{$<\mathbf{0 . 0 1}$} \\
\hline & Range 42.6-86.2 & Range 49.9-92.0 & Range 42.9-83.8 & \\
\hline \multicolumn{5}{|l|}{ Race } \\
\hline Other $(N=73)$ & $33 \%$ & $36 \%$ & $32 \%$ & \multirow[t]{2}{*}{0.26} \\
\hline White $(N=227)$ & $25 \%$ & $34 \%$ & $41 \%$ & \\
\hline \multicolumn{5}{|l|}{ Marital status } \\
\hline Other $(N=95)$ & $35 \%$ & $36 \%$ & $30 \%$ & \multirow[t]{2}{*}{0.05} \\
\hline Married $(N=167)$ & $24 \%$ & $32 \%$ & $44 \%$ & \\
\hline \multicolumn{5}{|l|}{ Currently employed } \\
\hline No $(N=136)$ & $27 \%$ & $32 \%$ & $41 \%$ & \multirow[t]{2}{*}{0.99} \\
\hline Yes $(N=11)$ & $28 \%$ & $32 \%$ & $40 \%$ & \\
\hline \multicolumn{5}{|l|}{ BMI category at diagnosis } \\
\hline Normal $(18.5$ to $<25)(N=94)$ & $21 \%$ & $44 \%$ & $35 \%$ & \multirow[t]{4}{*}{$<0.01$} \\
\hline Overweight $(25$ to $<30)(N=80)$ & $29 \%$ & $28 \%$ & $43 \%$ & \\
\hline Obese I $(30$ to $<35)(N=59)$ & $19 \%$ & $39 \%$ & $42 \%$ & \\
\hline Obese II $(\geq 35)(N=52)$ & $46 \%$ & $15 \%$ & $39 \%$ & \\
\hline \multirow[t]{2}{*}{ Weight at start of ET } & 83.9 (SD 21.4) & 72.8 (SD 16.5) & $75.6(\mathrm{SD} 15.7)$ & \multirow[t]{2}{*}{$<0.01$} \\
\hline & Range 46.8-149.0 & Range 42.7-124.5 & Range $42.3-120.9$ & \\
\hline \multirow[t]{2}{*}{ Weight change $(\mathrm{kg})$ between diagnosis and start of ET } & $0.04(\mathrm{SD} 3.2)$ & $-0.6(\mathrm{SD} 3.1)$ & $-2.9(\mathrm{SD} 4.9)$ & \multirow[t]{2}{*}{$<0.01$} \\
\hline & Range 8.5-9.4 & Range 15.7-13.2 & Range -19.9 to 6.5 & \\
\hline \multicolumn{5}{|l|}{ Hormone replacement therapy } \\
\hline Yes $(N=117)$ & $26 \%$ & $37 \%$ & $38 \%$ & \multirow[t]{4}{*}{0.94} \\
\hline No $(N=89)$ & $28 \%$ & $36 \%$ & $36 \%$ & \\
\hline \multirow[t]{2}{*}{ Total number of comorbidities } & $2.5(\mathrm{SD} 1.6)$ & $2.1(\mathrm{SD} 1.5)$ & 2.3 (SD 1.6) & \\
\hline & Range $0.0-6.0$ & Range $0.0-6.0$ & Range $0.0-6.0$ & \\
\hline $0-1$ comorbidities $(N=103)$ & $23 \%$ & $38 \%$ & $39 \%$ & \multirow[t]{3}{*}{0.49} \\
\hline $2-3$ comorbidities $(N=138)$ & $26 \%$ & $34 \%$ & $40 \%$ & \\
\hline$\geq 4$ comorbidities $(N=59)$ & $36 \%$ & $27 \%$ & $37 \%$ & \\
\hline \multicolumn{5}{|l|}{ Stage } \\
\hline Stage $0(N=5)$ & $60 \%$ & $20 \%$ & $20 \%$ & \multirow[t]{4}{*}{$<0.01$} \\
\hline Stage I $(N=127)$ & $36 \%$ & $34 \%$ & $30 \%$ & \\
\hline Stage II $(N=108)$ & $24 \%$ & $33 \%$ & $43 \%$ & \\
\hline Stage III $(N=52)$ & $10 \%$ & $31 \%$ & $60 \%$ & \\
\hline \multicolumn{5}{|l|}{ Grade } \\
\hline $1(N=77)$ & $35 \%$ & $30 \%$ & $35 \%$ & 0.27 \\
\hline $2(N=124)$ & $28 \%$ & $33 \%$ & $39 \%$ & \\
\hline $3(N=83)$ & $19 \%$ & $39 \%$ & $42 \%$ & \\
\hline Estrogen receptor & & & & \\
\hline Positive $(N=294)$ & $28 \%$ & $34 \%$ & $39 \%$ & 0.38 \\
\hline Negative $(N=5)$ & $0 \%$ & $60 \%$ & $40 \%$ & \\
\hline Progesterone receptor & & & & \\
\hline Positive $(N=248)$ & $27 \%$ & $36 \%$ & $36 \%$ & 0.07 \\
\hline Negative $(N=48)$ & $27 \%$ & $21 \%$ & $52 \%$ & \\
\hline HER2 & & & & \\
\hline Positive $(N=35)$ & $30 \%$ & $34 \%$ & $36 \%$ & 0.03 \\
\hline Negative $(N=250)$ & $11 \%$ & $34 \%$ & $54 \%$ & \\
\hline
\end{tabular}




\begin{tabular}{|c|c|c|c|c|}
\hline & \multicolumn{3}{|c|}{ Weight status after 2 years on endocrine treatment (ET) } & \multirow[t]{2}{*}{$p$ value } \\
\hline & $\begin{array}{l}>2 \mathrm{~kg} \text { weight loss } \\
(N=81,27 \%)\end{array}$ & $\begin{array}{l}\text { Stable weight } \\
(N=102,34 \%)\end{array}$ & $\begin{array}{l}>2 \mathrm{~kg} \text { weight gain } \\
(N=117,39 \%)\end{array}$ & \\
\hline \multicolumn{5}{|l|}{ Surgery } \\
\hline Lumpectomy $(N=169)$ & $32.5 \%$ & $35.5 \%$ & $32 \%$ & \multirow[t]{2}{*}{$<0.01$} \\
\hline Mastectomy $(N=128)$ & $20 \%$ & $32 \%$ & $48 \%$ & \\
\hline \multicolumn{5}{|l|}{ Axillary surgery } \\
\hline Axillary dissection $(N=92)$ & $12 \%$ & $33 \%$ & $55 \%$ & \multirow[t]{2}{*}{$<0.01$} \\
\hline Sentinel biopsy $(N=190)$ & $34 \%$ & $33 \%$ & $33 \%$ & \\
\hline \multicolumn{5}{|l|}{ Chemotherapy } \\
\hline None $(N=152)$ & $36 \%$ & $38 \%$ & $27 \%$ & \multirow[t]{3}{*}{$<0.01$} \\
\hline Neoadjuvant $(N=76)$ & $8 \%$ & $30 \%$ & $62 \%$ & \\
\hline Adjuvant $(N=72)$ & $29 \%$ & $31 \%$ & $40 \%$ & \\
\hline \multicolumn{5}{|l|}{ Radiation } \\
\hline Yes $(N=203)$ & $26 \%$ & $33 \%$ & $41 \%$ & \multirow[t]{2}{*}{0.46} \\
\hline No $(N=97)$ & $30 \%$ & $36 \%$ & $34 \%$ & \\
\hline \multicolumn{5}{|l|}{ Endocrine treatment (ET) } \\
\hline Tamoxifen only $(N=43)$ & $23 \%$ & $42 \%$ & $35 \%$ & \multirow[t]{6}{*}{0.16} \\
\hline Exemestane only $(N=8)$ & $38 \%$ & $0 \%$ & $63 \%$ & \\
\hline Anastrozole only $(N=113)$ & $32 \%$ & $32 \%$ & $36 \%$ & \\
\hline Letrozole only $(N=57)$ & $18 \%$ & $37 \%$ & $46 \%$ & \\
\hline More than one $\mathrm{AI}(N=40)$ & $23 \%$ & $28 \%$ & $50 \%$ & \\
\hline Tamoxifen and $\mathrm{AI}(N=39)$ & $33 \%$ & $41 \%$ & $26 \%$ & \\
\hline Time from breast cancer diagnosis to start of ET (years) & 0.41 & 0.44 & 0.50 & 0.13 \\
\hline
\end{tabular}

Values in bold are statistically significant

$B M I$ body mass index, $\mathrm{kg}$ kilograms, $S D$ standard deviation, $H E R 2$ human epidermal growth factor receptor 2, AI aromatase inhibitor

BC diagnosis and start of ET was associated with weight gain during $\mathrm{ET} \mathrm{RR}=0.98(0.97,0.99)(p<0.0001)$. No significant differences based on ET were observed.

\section{Adjusted relative risk for weight gain}

To avoid multicollinearity among multiple measures for BC tumor severity (stage, PR, HER2) and treatment (breast surgery, node surgery, chemotherapy), we ran separate multivariable models of each treatment type adjusted for BC stage: In the surgery model, mastectomy $\mathrm{RR}=1.30(0.96,1.75)$ $(p=0.09)$ and stage $\mathrm{RR}=1.32(1.10,1.59)(p=0.002)$; In the node surgery model, axillary node dissection $\mathrm{RR}=1.36$ $(0.97,1.91)(p=0.08)$ and stage $\mathrm{RR}=1.24(1.00,1.54)$ $(p=0.05) ; \quad$ In the chemotherapy model, adjuvant chemotherapy $\mathrm{RR}=1.35(0.90,2.03)(p=0.15)$, neoadjuvant chemotherapy $\mathrm{RR}=1.95(1.29,2.93)(p=0.002)$, and stage $\mathrm{RR}=1.12(0.91,1.39)(p=0.29)$.

\section{Sub-analysis of weight loss prior to ET}

Weight loss between $\mathrm{BC}$ diagnosis and start of ET was associated with $>2 \mathrm{~kg}$ weight gain during ET. To investigate this further, we conducted a sub-analysis to evaluate which patient characteristics were associated with losing $>2 \mathrm{~kg}$ prior to ET. Patients with higher BC stage (22 vs 32 vs $47 \%, p<0.01$ ), increasing tumor grade ( 20 vs 30 vs $39 \%, p=0.05$ ), who received mastectomy ( 37 vs $25 \%$, $p=0.04)$, axillary node dissection (42 vs $25 \%, p<0.01$ ), and chemotherapy (43 vs $17 \%, p<0.0001$ ), were all significantly more likely to lose $>2 \mathrm{~kg}$ prior to ET.

\section{Discussion}

We conducted a retrospective cohort study to investigate a question of concern to both BC survivors and their oncology providers: is weight gain associated with taking ET in early-stage BC. The weight data evaluated in our study were routinely collected by nursing staff during clinic visits in the first 2 years of ET. We report that being on ET is not necessarily associated with weight gain in postmenopausal BC survivors; in fact, a majority of survivors in our sample $(61 \%)$ maintained their weight or lost weight within this timeframe. However, $39 \%$ of the women in our sample did gain $>2 \mathrm{~kg}$ during the first 2 years of ET. 
Fig. 1 Histogram of weight change ( $2 \mathrm{~kg}$ increments) during 2 years of endocrine treatment $(\%)$

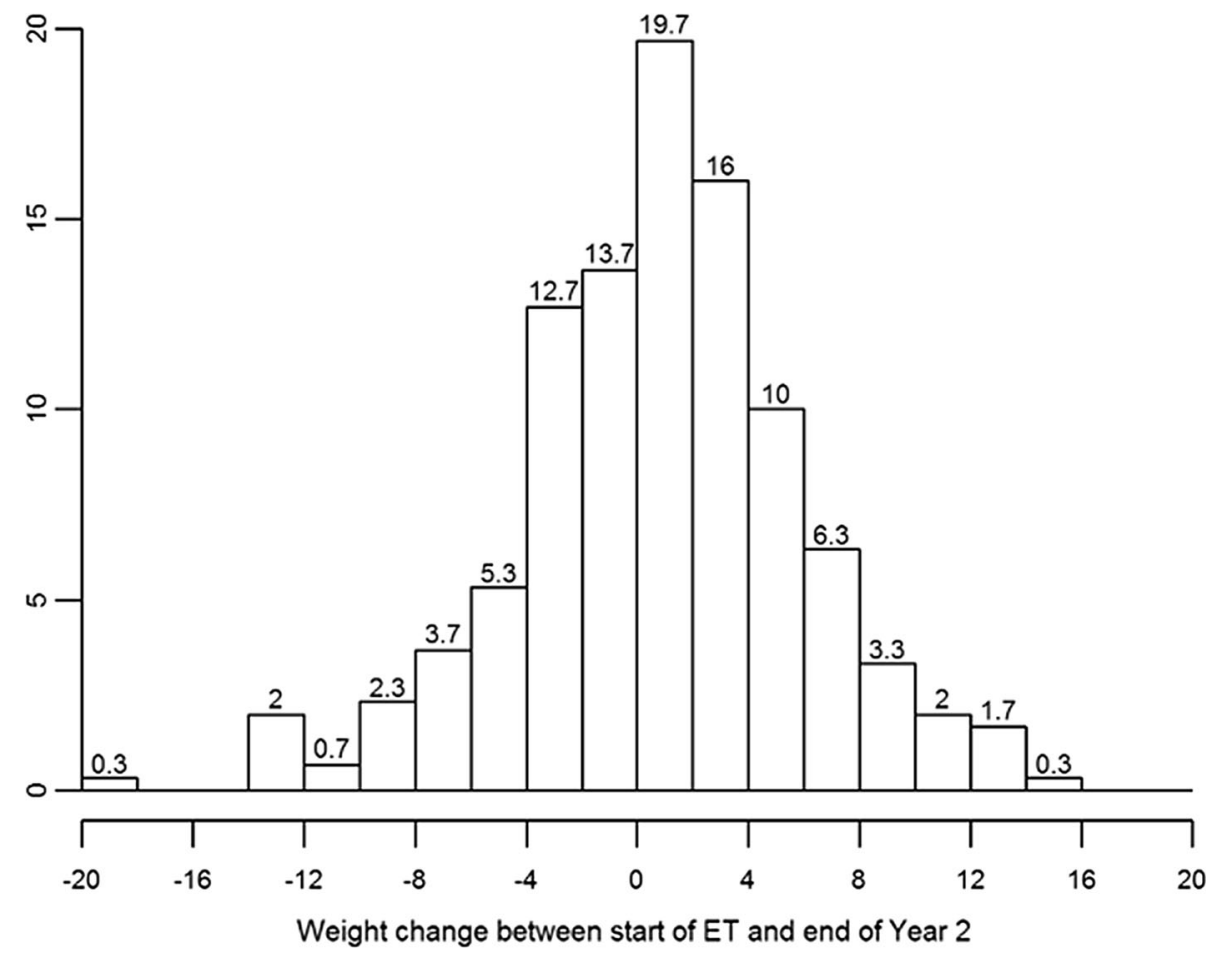

Fig. 2 Participants grouped by Body Mass Index at breast cancer diagnosis and weight change during endocrine treatment $(\%)$

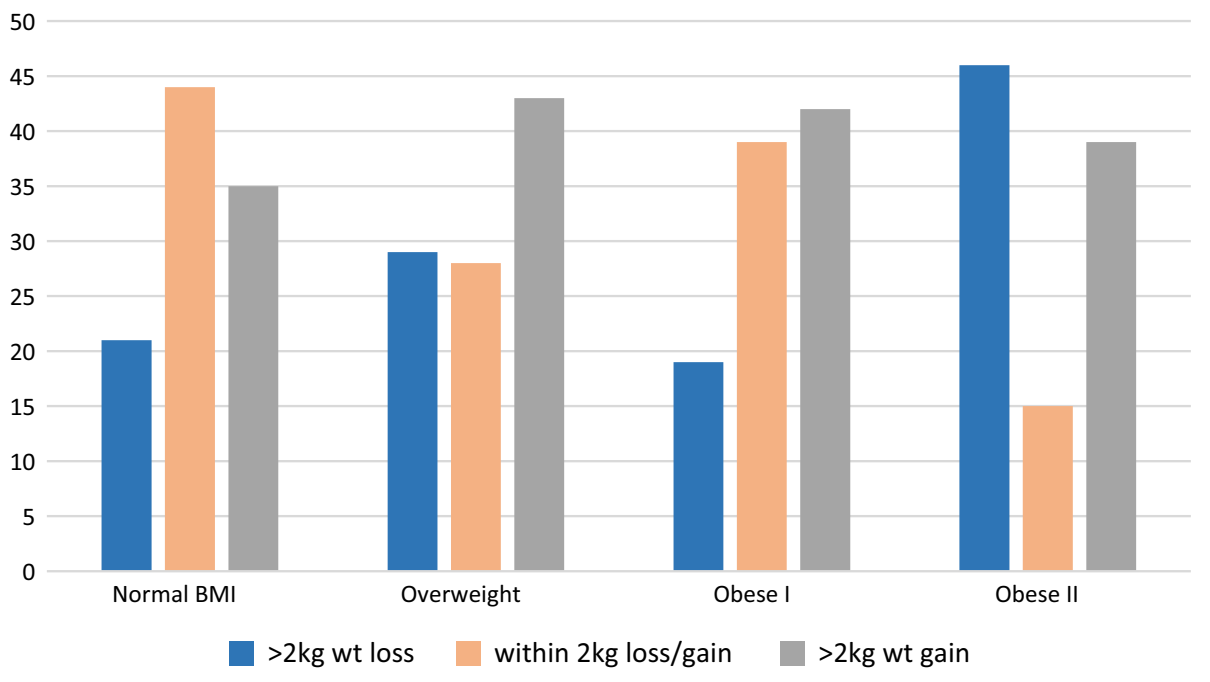

We found no differences in weight trajectories among the different ETs (tamoxifen and various AIs). Weight gain was primarily associated with higher disease stage and more intense treatment, with higher proportions of weight gain seen in women diagnosed with Stage II or III, PRpositive or HER2-positive tumor, having a mastectomy, undergoing axillary node dissection, receiving chemotherapy, and especially receiving neoadjuvant chemotherapy.

The use of EMR data for our retrospective cohort study had several advantages. One is the accuracy and details available in oncology provider and other clinical personnel notes describing BC diagnosis and treatment. Data were entered by clinical personnel into the EMR at the moment the data were gathered or shortly thereafter; no data were entered on a recall basis. For our study, diagnosis and treatment data were cross-checked throughout a patient's record to reduce the possibility of data misclassification. Variables analyzed in our study were for the same purposes as they were entered into the EMR; no new variables were implied or calculated. A further advantage of EMR data is the collection of weight data over time that was independently assessed by nursing staff using standardized clinic procedures, rather than self-reported weight. We did not have independent verification of ET adherence; however, evidence in clinician notes of ET non-adherence or discontinuation was a criterion for exclusion from the study. 
Table 3 Relative risk for gaining $>2 \mathrm{~kg}$ versus not gaining $>2 \mathrm{~kg}$ (stable weight or weight loss)

\begin{tabular}{|c|c|c|}
\hline Variable & Relative risk $(95 \% \mathrm{CI})$ & $p$ value \\
\hline Age at diagnosis & $0.98(0.96,0.99)$ & 0.002 \\
\hline \multicolumn{3}{|l|}{ Race } \\
\hline Other $(N=73)$ & Ref & \\
\hline White $(N=227)$ & $1.31(0.91,1.91)$ & 0.15 \\
\hline \multicolumn{3}{|l|}{ Marital status } \\
\hline Other $(N=95)$ & Ref & \\
\hline Married $(N=167)$ & $1.48(1.04,2.12)$ & $\mathbf{0 . 0 3}$ \\
\hline \multicolumn{3}{|l|}{ Currently employed } \\
\hline No $(N=136)$ & Ref & \\
\hline Yes $(N=11)$ & $0.97(0.72,1.32)$ & 0.85 \\
\hline \multicolumn{3}{|l|}{ BMI at diagnosis } \\
\hline Normal $(18.5$ to $<25)(N=94)$ & Ref & \\
\hline Overweight $(25$ to $<30)(N=80)$ & $1.21(0.83,1.76)$ & 0.32 \\
\hline Obese I $(30$ to $<35)(N=59)$ & $1.21(0.80,1.81)$ & 0.36 \\
\hline Obese II $(\geq 35)(N=52)$ & $1.10(0.71,1.70)$ & 0.68 \\
\hline Weight change $(\mathrm{kg})$ between diagnosis and start of ET & $0.98(0.97,0.99)$ & $<0.0001$ \\
\hline Weight at start of ET & $1.00(0.99,1.00)$ & 0.36 \\
\hline \multicolumn{3}{|l|}{ Hormone replacement therapy } \\
\hline Yes $(N=117)$ & $1.05(0.73,1.50)$ & 0.81 \\
\hline No $(N=89)$ & Ref & \\
\hline \multicolumn{3}{|l|}{ Total number of comorbidities } \\
\hline $0-1$ comorbidities $(N=103)$ & Ref & \\
\hline $2-3$ comorbidities $(N=138)$ & $1.03(0.75,1.41)$ & 0.87 \\
\hline$\geq 4$ comorbidities $(N=59)$ & $0.96(0.64,1.45)$ & 0.85 \\
\hline \multicolumn{3}{|l|}{ Stage } \\
\hline Stage $0(N=5)$ & $0.67(0.11,3.94)$ & 0.66 \\
\hline Stage I $(N=127)$ & Ref & \\
\hline Stage II $(N=108)$ & $1.42(1.01,2.01)$ & 0.04 \\
\hline Stage III $(N=52)$ & $1.99(1.41,2.82)$ & 0.0001 \\
\hline \multicolumn{3}{|l|}{ Grade } \\
\hline $1(N=77)$ & Ref & \\
\hline $2(N=124)$ & $1.10(0.76,1.61)$ & 0.61 \\
\hline $3(N=83)$ & $1.20(0.81,1.78)$ & 0.36 \\
\hline \multicolumn{3}{|l|}{ Estrogen receptor } \\
\hline Positive $(N=294)$ & $0.97(0.33,2.86)$ & 0.96 \\
\hline Negative $(N=5)$ & Ref & \\
\hline \multicolumn{3}{|l|}{ Progesterone receptor } \\
\hline Positive $(N=248)$ & $0.70(0.51,0.96)$ & 0.03 \\
\hline Negative $(N=48)$ & Ref & \\
\hline \multicolumn{3}{|l|}{ HER2 } \\
\hline Positive $(N=35)$ & $1.51(1.07,2.13)$ & 0.02 \\
\hline Negative $(N=250)$ & Ref & \\
\hline \multicolumn{3}{|l|}{ Surgery } \\
\hline Lumpectomy $(N=169)$ & Ref & \\
\hline Mastectomy $(N=128)$ & $1.49(1.12,1.98)$ & 0.006 \\
\hline \multicolumn{3}{|l|}{ Axillary surgery } \\
\hline Axillary dissection $(N=(92)$ & $1.67(1.27,2.20)$ & 0.0002 \\
\hline Sentinel biopsy $(N=190)$ & Ref & \\
\hline
\end{tabular}


Table 3 continued

\begin{tabular}{|c|c|c|}
\hline Variable & Relative risk (95\% CI) & $p$ value \\
\hline \multicolumn{3}{|l|}{ Chemotherapy } \\
\hline None $(N=152)$ & Ref & \\
\hline Neoadjuvant $(N=76)$ & $2.29(1.67,3.14)$ & $<0.0001$ \\
\hline Adjuvant $(N=72)$ & $1.49(1.02,2.19)$ & 0.04 \\
\hline \multicolumn{3}{|l|}{ Radiation } \\
\hline Yes $(N=203)$ & $1.22(0.88,1.68)$ & 0.23 \\
\hline No $(N=97)$ & Ref & \\
\hline \multicolumn{3}{|l|}{ Endocrine treatment (ET) } \\
\hline Tamoxifen only $(N=43)$ & Ref & \\
\hline Exemestane only $(N=8)$ & $1.79(0.91,3.52)$ & 0.09 \\
\hline Anastrozole only $(N=113)$ & $1.04(0.65,1.67)$ & 0.87 \\
\hline Letrozole only $(N=57)$ & $1.31(0.80,2.15)$ & 0.29 \\
\hline More than one $\mathrm{AI}(N=40)$ & $1.43(0.86,2.39)$ & 0.17 \\
\hline Tamoxifen and $\mathrm{AI}(N=39)$ & $0.74(0.38,1.44)$ & 0.37 \\
\hline Time from breast cancer diagnosis to start of ET & $0.22(0.01,0.43)$ & 0.04 \\
\hline
\end{tabular}

Relative risks in bold are statistically significant

And, the first 2 years of ET are a time period when ET adherence and continuation as prescribed are among the topics for provider-patient discussions during routinely scheduled clinic visits.

An important limitation of our study is that the EMR used in our study did not include high-quality data on other factors that could affect weight trajectories, such as information on the patient's engagement in physical activity and healthy eating behavior. Our EMR also did not include consistent collection of data on depression, anxiety, fatigue, pain, and other symptoms that may directly (or indirectly through reduced physical activity and/or alterations in eating habits) affect weight changes over time. All of these variables are essential for understanding weight trajectories in BC survivors and in determining how to intervene when variables are modifiable.

White women comprised a majority of the women in our study; however, the proportion in our sample (76\%) is in line with the general population of BC survivors diagnosed with hormone receptor-positive tumors. With regard to HR-positive/HER2-negative tumors, the incidence rate of $92.7(92.7,92.8)$ in non-Hispanic white women is higher than the rate of $74.4(74.2,74.6)$ in non-Hispanic black women [32]. A study of women presenting with stage I-III BC similarly showed a significant difference between black $(59.6 \%)$ as compared to white women $(76.1 \%)$ with regard to HR-positive tumors $(p<0.001)$ [66]. That study also reported a higher proportion of high-grade tumors in black $(64.6 \%)$ as compared to white $(43.0 \%)$ women $(p<0.001)$ [66], which may have contributed to the exclusion of some black women from our study due to $\mathrm{BC}$ recurrence or metastasis within the first years since BC diagnosis. With regard to postmenopausal women, non-Hispanic black women age 50 and above are more likely to have HRnegative tumors compared to all other racial and ethnic groups [67]. Thus, we believe our sample is generalizable to the overall population of postmenopausal BC survivors with early-stage hormone receptor-positive tumors.

Our study contributes to the literature pertaining to weight gain in BC survivors and specifically to the very limited literature pertaining to weight change during ET. We identified only one other study in which the sample consisted primarily of postmenopausal BC survivors, which reported $2-5 \mathrm{~kg}$ weight gain within the first year in $27 \%$ of women on anastrozole and $27 \%$ of women on tamoxifen [58]. Further studies are needed to compare different types of ET within a larger sample of BC survivors and to investigate the myriad additional factors that may contribute to clinically significant weight gain in survivorship. It is important that future studies focus on longitudinal weight trajectories with clearly stated start and end dates, to facilitate comparisons of findings across studies. It is also important for future studies to include amount of weight change in kilograms or pounds, and describe patient characteristics associated with weight gain loss or stable weight rather than just mean weight change.

While it is reassuring that a majority of the postmenopausal BC survivors in our sample did not gain $>2 \mathrm{~kg}$ during ET, a sizeable proportion did gain weight. The associations with disease severity and related treatment that 
we report in our study are suggestive for oncology providers as to which survivors are most likely to gain clinically significant weight during ET. When the BC treatment plan is first discussed, when the ET is started, and during regularly scheduled clinic visits in the first 2 years of ET are the time points in the cancer care continuum that present opportunities for oncology provider-patient communication about the importance of weight management. Our own research shows that $\mathrm{BC}$ patients are interested in having timely communications about ET side effects [68] and that it is feasible for medical oncology providers to have communications with their patients about healthy behaviors such as physical activity [69]. For many women, the $\mathrm{BC}$ diagnosis can be an important "teachable moment" [70-73] where oncology providers can impart information and encouragement to their patients regarding healthy behaviors that promote wellness and quality of life and, potentially, improved prognosis and survival [74-76].

Acknowledgements This study was supported by the Breast Cancer Research Foundation of New York and the UNC Lineberger Comprehensive Cancer Center/University Cancer Research Fund. Dr. Shachar's fellowship at UNC was supported by the Friends of
Rambam Medical Center and The J\&G Zukier Medical Fund Donation, Haifa, Israel.

Funding This study was funded in part by the Breast Cancer Research Foundation of New York, and UNC Lineberger Comprehensive Cancer Center/University Cancer Research Fund.

\section{Compliance with ethical standards}

Conflict of interest The authors declare they have no conflicts of interest.

Ethical approval All procedures performed in studies involving human participants were in accordance with the ethical standards of the institutional and/or national research committee and with the 1964 Helsinki declaration and its later amendments or comparable ethical standards.

Informed consent This study did not entail direct contact with humans and therefore did not entail obtaining informed consent.

\section{Appendix}

See Fig. 3.

Fig. 3 Flow diagram

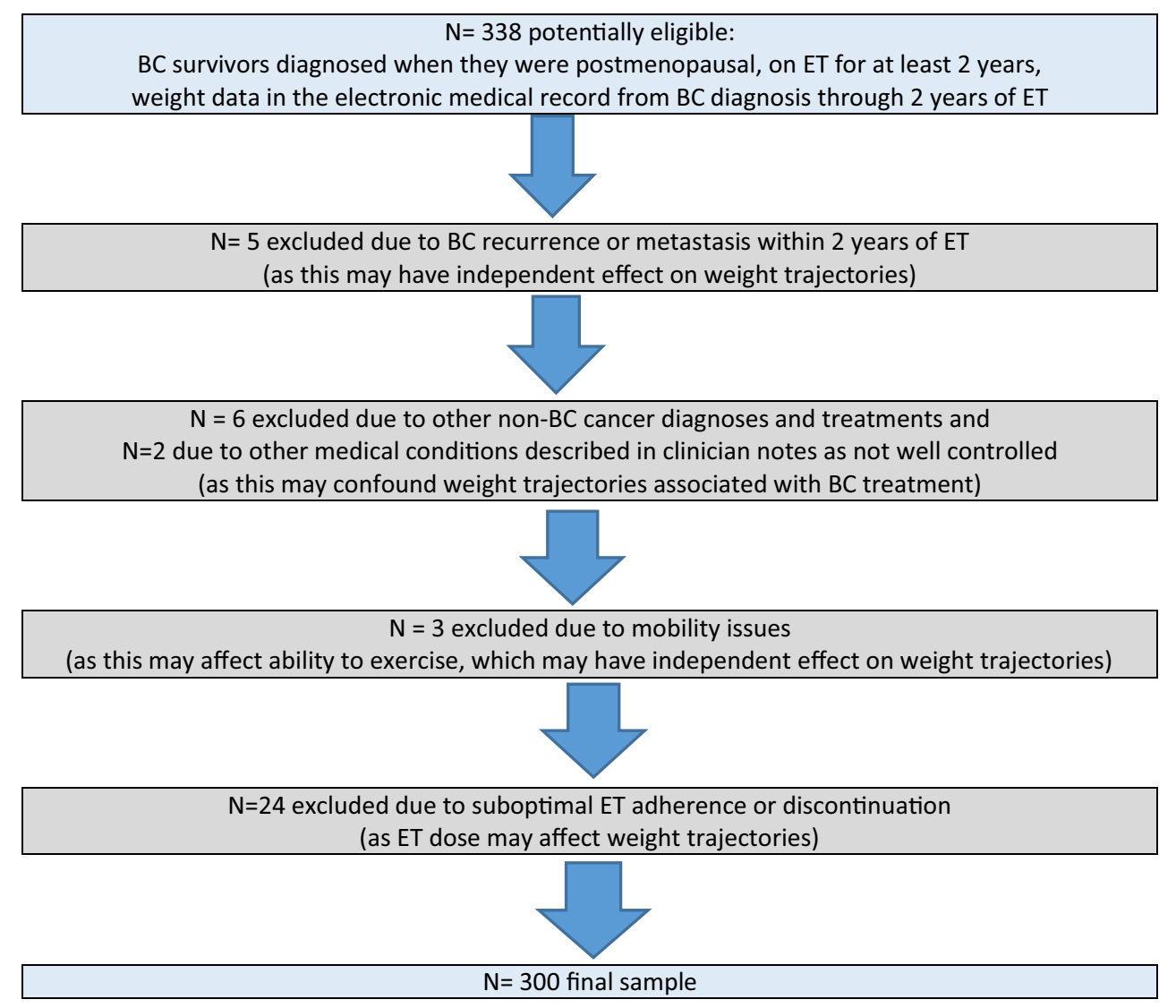




\section{References}

1. Playdon MC, Bracken MB, Sanft TB, Ligibel JA, Harrigan M, Irwin ML (2015) Weight gain after breast cancer diagnosis and all-cause mortality: systematic review and meta-analysis. J Natl Cancer Inst 107(12):275

2. Ligibel JA, Alfano CM, Courneya KS et al (2014) American Society of Clinical Oncology position statement on obesity and cancer. J Clin Oncol 32(31):3568-3574

3. Ligibel JA, Alfano CM, Hershman D et al (2015) Recommendations for obesity clinical trials in cancer survivors: American Society of Clinical Oncology Statement. J Clin Oncol 33(33):3961-3967

4. Denlinger CS, Ligibel JA, Are M et al (2014) Survivorship: nutrition and weight management, Version 2.2014. Clinical practice guidelines in oncology. J Natl Compr Canc Netw 12(10):1396-1406

5. Anderson AS, Key TJ, Norat T et al (2015) European code against cancer 4th edition: obesity, body fatness and cancer. Cancer Epidemiol 39(Suppl 1):S34-S45

6. Alfano CM, Bluethmann SM, Tesauro G et al (2016) NCI Funding trends and priorities in physical activity and energy balance research among cancer survivors. J Natl Cancer Inst. doi:10.1093/jnci/djv285

7. Ballard-Barbash R, Siddiqi SM, Berrigan DA, Ross SA, Nebeling LC, Dowling EC (2013) Trends in research on energy balance supported by the National Cancer Institute. Am J Prev Med 44(4):416-423

8. Alfano CM, Molfino A, Muscaritoli M (2013) Interventions to promote energy balance and cancer survivorship: priorities for research and care. Cancer 119(Suppl 11):2143-2150

9. Rock CL, Doyle C, Demark-Wahnefried W et al (2012) Nutrition and physical activity guidelines for cancer survivors. CA Cancer J Clin 62(4):243-274

10. Schmitz KH, Courneya KS, Matthews C, Demark-Wahnefried W, Galvao DA et al (2010) American college of sports medicine roundtable on exercise guidelines for cancer survivors. Med Sci Sports Exerc 42(7):1409-1426

11. Kushi LH, Doyle C, McCullough M et al (2012) American Cancer Society guidelines on nutrition and physical activity for cancer prevention: reducing the risk of cancer with healthy food choices and physical activity. CA Cancer J Clin 62(1):30-67

12. Demark-Wahnefried W, Peterson B, McBride C, Lipkus I, Clipp E (2000) Current health behaviors and readiness to pursue lifestyle changes among men and women diagnosed with early stage prostate and breast carcinomas. Cancer 88(3):674-684

13. Harvie M (2010) The importance of controlling body weight after a diagnosis of breast cancer: the role of diet and exercise in breast cancer patient management. In: Saxton J, Daley A (eds) Exercise and cancer survivorship: impact on health outcomes and quality of life. Springer, New York

14. Key TJ, Appleby PN, Reeves GK, Roddam A, Dorgan JF et al (2003) Body mass index, serum sex hormones, and breast cancer risk in postmenopausal women. $\mathrm{N}$ Natl Cancer Inst 95(16): 1218-1226

15. Cheraghi Z, Poorolajal J, Hashem T, Esmailnasab N, Doosti Irani A (2012) Effect of body mass index on breast cancer during premenopausal and postmenopausal periods: a meta-analysis. PLoS ONE 7(12):e51446

16. Keum N, Greenwood DC, Lee DH et al (2015) Adult weight gain and adiposity-related cancers: a dose-response meta-analysis of prospective observational studies. J Natl Cancer Inst. doi:10. 1093/jnci/dju428
17. La Vecchia C, Giordano SH, Hortobagyi GN, Chabner B (2011) Overweight, obesity, diabetes, and risk of breast cancer: interlocking pieces of the puzzle. Oncologist 16(6):726-729

18. Xia X, Chen W, Li J et al (2014) Body mass index and risk of breast cancer: a nonlinear dose-response meta-analysis of prospective studies. Sci Rep 4:7480

19. Coughlin SS, Smith SA (2015) The insulin-like growth factor axis, adipokines, physical activity, and obesity in relation to breast cancer incidence and recurrence. Cancer Clin Oncol 4(2):24-31

20. Petrelli JM, Calle EE, Rodriguez C, Thun MJ (2002) Body mass index, height, and postmenopausal breast cancer mortality in a prospective cohort of US women. Cancer Causes Control 13(4):325-332

21. Nechuta S, Chen WY, Cai H et al (2016) A pooled analysis of post-diagnosis lifestyle factors in association with late estrogenreceptor-positive breast cancer prognosis. Int $\mathrm{J}$ Cancer 138(9):2088-2097

22. Bradshaw PT, Ibrahim JG, Stevens J, Cleveland RJ, Abrahamson PE et al (2012) Postdiagnosis change in bodyweight and survival after breast cancer diagnosis. Epidemiology 23(2):320-327

23. Niraula S, Ocana A, Ennis M, Goodwin PJ (2012) Body size and breast cancer prognosis in relation to hormone receptor and menopausal status: a meta-analysis. Breast Cancer Res Treat 134(2):769-781

24. Dixon JK, Moritz DA, Baker FL (1978) Breast cancer and weight gain: an unexpected finding. Oncol Nurs Forum 5(3):5-7

25. Levine EG, Raczynski JM, Carpenter JT (1991) Weight gain with breast cancer adjuvant treatment. Cancer 67(7):1954-1959

26. Demark-Wahnefried W, Winer EP, Rimer BK (1993) Why women gain weight with adjuvant chemotherapy for breast cancer. J Clin Oncol 11(7):1418-1429

27. Anderson WF, Pfeiffer RM, Dores GM, Sherman ME (2006) Comparison of age distribution patterns for different histopathologic types of breast carcinoma. Cancer Epidemiol Biomark Prev 15(10):1899-1905

28. Benz CC (2008) Impact of aging on the biology of aging. Crit Rev Oncol Hematol 66(1):65-74

29. Howlader N, Altekruse SF, Li CI et al (2014) US incidence of breast cancer subtypes defined by joint hormone receptor and HER2 status. J Natl Cancer Inst. doi:10.1093/jnci/dju055

30. Clark GM, Osborne CK, McGuire WL (1984) Correlations between estrogen receptor, progesterone receptor, and patient characteristics in human breast cancer. J Clin Oncol 2:1102-1109

31. Jenkins EO, Deal AM, Anders CK et al (2014) Age-specific changes in intrinsic breast cancer subtypes: a focus on older women. Oncologist 19(10):1076-1083

32. Kohler BA, Sherman RL, Howlader N et al (2015) Annual report to the nation on the status of cancer, 1975-2011, featuring incidence of breast cancer subtypes by race/ethnicity, poverty, and state. J Natl Cancer Inst 107(6):djv048

33. Dunnwald LK, Rossing MA, Li CI (2007) Hormone receptor status, tumor characteristics, and prognosis: a prospective cohort of breast cancer patients. Breast Cancer Res 9(1):R6

34. Eppenberger-Castori S, Moore DH Jr, Thor AD et al (2002) Ageassociated biomarker profiles of human breast cancer. Int $\mathrm{J}$ Biochem Cell Biol 34(11):1318-1330

35. Taylor WC, Muss HB (2010) Recent advances: adjuvant therapy for older women with breast cancer. Cancer J 16(4):289-293

36. Schiavon G, Smith IE (2014) Status of adjuvant endocrine therapy for breast cancer. Breast Cancer Res 16(2):206

37. Dowsett M, Cuzick J, Ingle J et al (2010) Meta-analysis of breast cancer outcomes in adjuvant trials of aromatase inhibitors versus tamoxifen. J Clin Oncol 28(3):509-518 
38. Goss PE, Ingle JN, Pritchard KI, et al (2016) Extending aromatase-inhibitor adjuvant therapy to 10 years. $\mathrm{N}$ Engl $\mathrm{J}$ Med 375(3):209-219

39. Sestak I, Cuzick J, Sapunar F et al (2008) Risk factors for joint symptoms in patients enrolled in the ATAC trial: a retrospective, exploratory analysis. Lancet Oncol 9(9):866-872

40. Mao JJ, Chung A, Benton A et al (2013) Online discussion of drug side effects and discontinuation among breast cancer survivors. Pharmacoepidemiol Drug Saf 22(3):256-262

41. Aiello Bowles EJ, Boudreau DM, Chubak J et al (2012) Patientreported discontinuation of endocrine therapy and related adverse effects among women with early-stage breast cancer. J Oncol Pract 8(6):49-57

42. Malinovszky KM, Cameron D, Douglas S et al (2004) Breast cancer patients' experiences on endocrine therapy: monitoring with a checklist for patients on endocrine therapy (C-PET). Breast 13(5):363-368

43. Kadakia KC, Snyder CF, Kidwell KM et al (2016) Patient-reported outcomes and early discontinuation in aromatase inhibitortreated postmenopausal women with early stage breast cancer. Oncologist 21(5):539-546

44. Sestak I, Distler W, Forbes JF, Dowsett M, Howell A, Cuzick J (2010) Effect of body mass index on recurrences in tamoxifen and anastrozole treated women: an exploratory analysis from the ATAC trial. J Clin Oncol 28(21):3411-3415

45. Wolters R, Schwentner L, Regierer A, Wischnewsky M, Kreienberg R, Wöckel A (2012) Endocrine therapy in obese patients with primary breast cancer: another piece of evidence in an unfinished puzzle. Breast Cancer Res Treat 131(3): 925-931

46. Gnant M, Pfeiler G, Stoger H et al (2013) The predictive impact of body mass index on the efficacy of extended adjuvant endocrine treatment with anastrozole in postmenopausal patients with breast cancer: an analysis of the randomised ABCSG-6a trial. Br J Cancer 109(3):589-596

47. Pfeiler G, Stoger H, Dubsky P et al (2013) Efficacy of tamoxifen \pm aminoglutethimide in normal weight and overweight postmenopausal patients with hormone receptor-positive breast cancer: an analysis of 1509 patients of the ABCSG-06 trial. Br J Cancer 108(7):1408-1414

48. Ioannides SJ, Barlow PL, Elwood JM, Porter D (2014) Effect of obesity on aromatase inhibitor efficacy in postmenopausal, hormone receptor-positive breast cancer: a systematic review. Breast Cancer Res Treat 147(2):237-248

49. Caan BJ, Emond JA, Su HI et al (2012) Effect of postdiagnosis weight change on hot flash status among early-stage breast cancer survivors. J Clin Oncol 30(13):1492-1497

50. Su H, Sammel MD, Springer E, Freeman EW, DeMichele A, Mao JJ (2010) Weight gain is associated with increased risk of hot flashes in breast cancer survivors on aromatase inhibitors. Breast Cancer Res Treat 124(1):205-211

51. Young A, Weltzien E, Kwan M, Castillo A, Caan B, Kroenke CH (2014) Pre- to post-diagnosis weight change and associations with physical functional limitations in breast cancer survivors. J Cancer Surviv 8(4):539-547

52. Voskuil DW, van Nes JG, Junggeburt JM, van de Velde CJ, van Leeuwen FE, de Haes JC (2010) Maintenance of physical activity and body weight in relation to subsequent quality of life in postmenopausal breast cancer patients. Ann Oncol 21(10):2094-2101

53. Nyrop KA, Williams GR, Muss HB, Shachar SS (2016) Weight gain during adjuvant endocrine treatment for early-stage breast cancer: what is the evidence? Breast Cancer Res Treat 158(2):203-217

54. Gallicchio L, MacDonald R, Wood B, Rushovich E, Helzlsouer KJ (2012) Menopausal-type symptoms among breast cancer patients on aromatase inhibitor therapy. Climacteric 15(4):339-349

55. Heideman WH, Russell NS, Gundy C, Rookus MA, Voskuil DW (2009) The frequency, magnitude and timing of post-diagnosis body weight gain in Dutch breast cancer survivors. Eur J Cancer 45:119-126

56. van de Velde CJ, Rea D, Seynaeve C et al (2011) Adjuvant tamoxifen and exemestane in early breast cancer (TEAM): a randomised phase 3 trial. Lancet 377(9762):321-331

57. Fallowfield LJ, Kilburn LS, Langridge C et al (2012) Long-term assessment of quality of life in the intergroup exemestane study: 5 years post-randomisation. Br J Cancer 106(6):1062-1067

58. Sestak I, Harvie M, Howell A, Forbes JF, Dowsett M, Cuzick J (2012) Weight change associated with anastrozole and tamoxifen treatment in postmenopausal women with or at high risk of developing breast cancer. Breast Cancer Res Treat 134(2):727-734

59. Vassar M, Holzmann M (2013) The retrospective chart review: important methodological considerations. J Edu Eval Health Prof 10:12

60. Goodwin PJ, Ennis M, Pritchard KI, McCready D, Koo J et al (1999) Adjuvant treatment and onset of menopause predict weight gain after breast cancer diagnosis. J Clin Oncol 17(1):120-129

61. Hoskin PJ, Ashley S, Yarnold JR (1992) Weight gain after primary surgery for breast cancer-effect of tamoxifen. Breast Cancer Res Treat 22(2):129-132

62. Kritchevsky SB, Wilcosky TC, Morris DL, Truong KN, Tyroler HA (1991) Changes in plasma lipid and lipoprotein cholesterol and weight prior to the diagnosis of cancer. Cancer Res 51(12):3198-3203

63. McTiernan A, Sorensen B, Irwin ML et al (2007) Exercise effect on weight and body fat in men and women. Obesity (Silver Spring, Md.) 15(6):1496-1512

64. Myers CA, Slack T, Martin CK, Broyles ST, Heymsfield SB (2016) Change in obesity prevalence across the united states is influenced by recreational and healthcare contexts, food environments, and hispanic populations. PLoS ONE 11(2):e0148394

65. Harris PA, Taylor R, Thielke R, Payne J, Gonzalez N, Conde JG (2009) Research electronic data capture (REDCap) — a metadatadriven methodology and workflow process for providing translational research informatics support. $\mathrm{J}$ Biomed Inform 2009(42):377-381

66. Warner ET, Tamimi RM, Hughes ME et al (2015) Racial and ethnic differences in breast cancer survival: mediating effect of tumor characteristics and sociodemographic and treatment factors. J Clin Oncol 33(20):2254-2261

67. Hou N, Huo D (2013) A trend analysis of breast cancer incidence rates in the United States from 2000 to 2009 shows a recent increase. Breast Cancer Res Treat 138(2):633-641

68. Nyrop KA, Callahan LF, Rini C et al (2016) Aromatase inhibitor associated arthralgia: the importance of oncology provider-patient communication about side effects and potential management through physical activity. Support Care Cancer 24(6):2643-2650

69. Nyrop KA, Deal AM, Williams GR, Guerard EJ, Pergolotti M, Muss HB (2016) Physical activity communication between oncology providers and patients with early-stage breast, colon, or prostate cancer. Cancer 122(3):470-476

70. Demark-Wahnefried W, Aziz NM, Rowland JH, Pinto BM (2005) Riding the crest of the teachable moment: promoting longterm health after the diagnosis of cancer. J Clin Oncol 23(24):5814-5830

71. Rabin C (2009) Promoting lifestyle change among cancer survivors: when is the teachable moment? Am J Lifestyle Med 3:369

72. Anderson AS, Mackison D, Boath C, Steele R (2013) Promoting changes in diet and physical activity in breast and colorectal 
cancer screening settings: an unexplored opportunity for endorsing healthy behaviors. Cancer Prev Res (Phila) 6(3):165-172

73. Humpel N, Magee C, Jones SC (2007) The impact of a cancer diagnosis on the health behaviors of cancer survivors and their family and friends. Support Care Cancer 15(6):621-630

74. Wolin KY, Schwartz AL, Matthews CE, Courneya KS, Schmitz KH (2012) Implementing the exercise guidelines for cancer survivors. J Support Oncol 10(5):171-177
75. Santa Mina D, Alibhai SM, Matthew AG et al (2012) Exercise in clinical cancer care: a call to action and program development description. Curr Oncol 19(3):e136-e144

76. Ruiz-Casado A, Lucia A (2014) The time has come for oncologists to recommend physical activity to cancer survivors. Arch Exerc Health Dis 4(1):214-215 